\title{
Type 3 Error
}

National Cancer Institute

\section{Source}

National Cancer Institute. Type 3 Error. NCI Thesaurus. Code C142741.

In statistics, the probability of a Type 3 error, when one correctly rejects the null hypothesis, but the reason for rejecting it is incorrect. In the context of study interventions, this means that one incorrectly determines that the less useful treatment is more useful. 